\title{
Stress and Strain in an Orthopaedic Department on the Frontlines during the COVID-19 Pandemic: An Analysis of Burnout and the Factors Influencing It
}

\section{Dear Editor,}

Burnout is common among physicians and affects the quality of patient care. ${ }^{1,2}$ Preliminary studies have shown that the COVID-19 pandemic has negatively impacted the emotional and mental wellness of healthcare workers. ${ }^{3,4}$ Our original study aims to analyse the effect this pandemic has on burnout rates and possible reasons among all members of the orthopaedic department in Ng Teng Fong General Hospital, Singapore.

This is an anonymous, questionnaire-based, crosssectional study conducted prospectively in an orthopaedic department of a tertiary hospital during the COVID-19 pandemic. From 1 to 10 April 2020, all healthcare workers within the orthopaedic department were invited via an email blast to participate in a self-administered questionnaire.

The primary objective of this study is to discover the prevalence of burnout before and after the hospital's implementation of measures countering the COVID-19 pandemic. Our secondary objectives are to correlate demographic data with burnout, as well as identify the reasons for burnout.

Our selection of participants consisted of the entire orthopaedic department, including 58 doctors, 70 nurses, 24 administrative staff and 4 allied healthcare staff. The questionnaires were then distributed electronically.

Responses to each questionnaire item were collected in Google forms and tabulated using Microsoft Excel 2016. The questionnaire was divided into 3 sections that recorded: (1) participant demographics, (2) the Maslach Burnout Inventory (MBI) designed for Medical Personnel and (3) questions on factors influencing burnout.

The MBI is the most common validated tool used to measure physician burnout in the last 20 years. ${ }^{5,6}$ It is a 22-item survey that measures burnout on 3 separate subscales: depersonalisation (score range of $0-30$ ), emotional exhaustion (score range of 0-54) and low personal accomplishment (score range of 0-48). As for the reasons influencing burnout, we provided a list of possible reasons influencing burnout as well as a free response section. Participants were required to rate each reason from "not influencing burnout" to "significantly influencing burnout".
Quantitative data were analysed using SPSS software (version 26; SPSS Inc.). Associations between variables were evaluated using the chi-square test for categorical variables and paired t-test for continuous variables. Multivariate logistic regression analysis to identify parameters associated with the dependent variables (burnout) while adjusting for confounders was performed.

Of the 156 healthcare workers invited for this questionnaire survey, 117 (75\%) responded. Their mean age was 32.6 years old (SD 8.21), and there was a slightly higher proportion of females $(65.8 \%)$. Most of the participants (approximately 90\%) were nurses or doctors, which is consistent with the composition of the staff in the department. Among the doctors, there were equal numbers of senior and junior participants, with $50 \%$ of participants being senior residents and above. A chisquare goodness-of-fit test showed that there was no statistical significance between the study participants and the actual department demographics $(P=0.231)$.

Fifteen $(12.8 \%)$ of the participants indicated that they had extra appointments on top of their clinical duties (e.g. research coordinator, medical informatics), and $29(24.8 \%)$ participants indicated that they were posted to other frontline healthcare services amid the coronavirus pandemic.

Based on the MBI scores, the prevalence of healthcare staff experiencing burnout increased from 45 (38.5\%) before COVID-19 measures to 60 (51.3\%) after COVID-19 measures $(P<0.001)$. An analysis of MBI showed an increase in burnout scores in all 3 subscales, with the greatest proportion of increase in emotional exhaustion $(P=<0.001)$.

In the questionnaire, respondents were also required to indicate directly if burnout was worse amid the COVID-19 situation. Sixty-seven (57.3\%) of the respondents felt that burnout was worse, $44(37.6 \%)$ felt that burnout was unchanged, and $6(5.1 \%)$ felt that it was less.

After multivariate analysis, being an older healthcare participant was found to be protective against burnout, both before (odds ratio (OR) $0.84, P<0.01$ ) and after implementation of COVID-19 measures (OR 0.92, $P<0.01$ ) (Table 2). Being posted to other frontline 
healthcare resources during the coronavirus pandemic was also correlated with less burnout before COVID-19 measures (OR 0.32, $P=0.04$ ).

From our questionnaire, the top ranked reasons for burnout were: (1) fear of transmitting COVID-19 to family members/contacts, with $80(68.4 \%)$ respondents indicating that it "significantly influenced burnout";
(2) uncertainty of future, with 71 respondents $(60.7 \%)$ indicating that it "significantly influenced burnout"; (3) increase in workload, with 70 respondents $(59.8 \%)$ indicating no increase. In the free response section, $10(8.5 \%)$ indicated family issues (including finding caregivers for children and parents, children's schooling) as another factor that significantly influenced burnout.

Table 1. Association of demographic data with burnout

\begin{tabular}{|c|c|c|c|c|c|c|}
\hline \multirow[b]{2}{*}{ Independent variable } & \multicolumn{3}{|c|}{ Burnout before COVID-19 measures } & \multicolumn{3}{|c|}{ Burnout after COVID-19 measures } \\
\hline & Odds ratio $(\mathrm{SE})$ & CI 95\% & $P$ & Odds ratio (SE) & CI 95\% & $P$ \\
\hline Female sex & $1.37(0.54)$ & $0.48-3.93$ & 0.56 & $0.96(0.53)$ & $0.34-2.72$ & 0.94 \\
\hline \multicolumn{7}{|l|}{ Occupation } \\
\hline - Administrative staff & 1.00 & - & 0.67 & 1.00 & - & 0.79 \\
\hline - Nurse & $2.22(0.88)$ & $0.40-12.36$ & 0.36 & $2.22(0.77)$ & $0.37-8.33$ & 0.30 \\
\hline - Allied healthcare staff & $0.00(27459)$ & 0.00 & 0.99 & $0.00(27682)$ & 0.00 & 0.99 \\
\hline - Doctor & $3.45(1.00)$ & $0.49-24.23$ & 0.21 & $1.94(0.90)$ & $0.20-7.78$ & 0.46 \\
\hline $\begin{array}{l}\text { Extra appointments on top of } \\
\text { clinical duties }\end{array}$ & $1.32(0.67)$ & $0.35-4.92$ & 0.68 & $1.07(0.64)$ & $0.40-5.68$ & 0.91 \\
\hline $\begin{array}{l}\text { Posted to other frontline } \\
\text { services after COVID-19 } \\
\text { measures }\end{array}$ & $0.37(0.52)$ & $0.14-1.02$ & $0.05^{*}$ & $0.72(0.47)$ & $0.27-1.77$ & 0.48 \\
\hline
\end{tabular}

* Significant $P$ value $<0.05$

Table 2. Reasons that significantly influence burnout

\begin{tabular}{|c|c|}
\hline Proposed reasons & No. of respondents indicating "significantly influenced burnout" \\
\hline Fear of transmitting SARS-CoV-2 to family members/contacts & $80(68.4 \%)$ \\
\hline Uncertainty of future & $71(60.7 \%)$ \\
\hline Increase in workload & $70(59.8 \%)$ \\
\hline Uncertainty of promotion & $64(54.7 \%)$ \\
\hline Changes in workflows & $62(53.0 \%)$ \\
\hline Fear of contracting SARS-CoV-2 & $60(51.3 \%)$ \\
\hline Lack of training opportunities & $59(50.4 \%)$ \\
\hline $\begin{array}{l}\text { Stress from accumulating case-load } \\
\text { (as uncleared electives continue to increase) }\end{array}$ & $58(49.6 \%)$ \\
\hline External/non-work-related factors & $47(40.2 \%)$ \\
\hline
\end{tabular}


Substantial changes to the clinical workflow of the department affected not only the doctors, but all healthcare workers alike, and consisted of: (1) team segregation to reduce the possibility of cross contamination across key essential specialties, (2) reducing non-essential clinical and surgical activities to allow the capacity for handling expected increase in COVID-19 cases, and (3) rechanneling surplus resources to other frontline healthcare services.

Following the implementation of COVID-19 measures, burnout among the healthcare staff surveyed showed an increase from a substantial $38.5 \%$ to a high $51.3 \%$. Apart from age and whether a healthcare worker was posted out to other frontline healthcare services, gender, occupation and whether the participant was an orthopaedic resident or given extra appointments on top of clinical duties were not predictive of burnout.

Older healthcare workers were found to be at a lower risk of burnout in our study, perhaps due to the decrease in elective clinical workload and assignments given to them during the reorganisation. The mean age of the healthcare workers surveyed was 32.6. These essential workers were burdened with the responsibilities of caring and providing for their families, as well as challenged with the need to advance their career. It is no wonder factors such as "uncertainty of future", "uncertainty of promotion" and "lack of training opportunities" were also common reasons offered for burnout, beyond an increase in workload.

Among reasons cited by participants for experiencing burnout, $68.4 \%$ reported the fear of transmitting SARS$\mathrm{CoV}-2$ to family members/contacts, as opposed to $51.3 \%$ reporting fear of contracting SARS-CoV-2 themselves.

In addition, $59.8 \%$ of participants cited an increase in workload as a reason for experiencing burnout. With several members of our staff being posted out of the department to aid other frontline services as well as others on mandatory stay-home leave after being in contact with suspected COVID-19 cases, the remaining healthcare workers had to take up extra clinical duties. Implementing such changes in workflow also resulted in an increased workload, such as administrative staff being required to call up patients to reschedule clinic appointments and surgeries for non-urgent cases.

This study has several limitations. First, all data were collected at a single time point, 6 weeks after the COVID-19 measures were put in place. Therefore, burnout scores from before the implementation of COVID-19 measures could be affected by recall bias. Second, the dynamic nature of the outbreak resulted in numerous measures being rolled out in phases to combat the virus. This may have affected the quality of the data, and careful interpretation should be exercised. Thirdly, while the composition of the participants was similar to the department's demographics, the overall number of study participants was small and may limit its conclusions. Lastly, other demographic data that may also influence burnout, such as nationality, pre-existing medical conditions, marital status and socioeconomic status, which were not surveyed in this study and can be explored in future studies.

In conclusion, our original study shows that COVID-19 has had a significant impact on burnout in healthcare workers, with older workers having a lower risk of burnout. Most participants expressed concerns over transmitting the virus to family members/contacts, uncertainty of their future, and increase in workload. Today, given the evolving circumstances, the COVID-19 pandemic is likely to have more impact on healthcare workers in the long term. Future studies should look at strategies to manage stress and burnout in healthcare staff.

\section{Acknowledgements}

The authors would like to thank Mr Chiam Sheng Yeow (BE (Hon), National University of Singapore) for his invaluable assistance in data analysis.

\section{REFERENCES}

1. See KC, Lim TK, Kua EH, et al. Stress and Burnout among Physicians: Prevalence and Risk Factors in a Singaporean Internal Medicine Programme. Ann Acad Med Singap 2016;45:471-4.

2. Ng NS, Kwek EB. Are We Working Too Hard? - A Functional Scoring of Orthopaedic Surgeons. Ann Acad Med Singap 2016;45:212-4.

3. Goh SSN, Chia MYC. Anxiety and Morale in Front-Line Healthcare Workers During the Coronavirus Disease 2019 (COVID-19) Outbreak at the National Screening Centre in Singapore. Ann Acad Med Singap 2020;49:259-62.

4. Tan BY, Chew NW, Lee GK, et al. Psychological Impact of the COVID-19 Pandemic on Health Care Workers in Singapore. Ann Intern Med 2020;173:317-20.

5. Maslach C, Jackson SE, Leiter MP. Maslach Burnout Inventory Manual. 4th ed. Menlo Park, CA: Mind Garden Inc, 2016.

6. Rotenstein LS, Torre M, Ramos MA, et al. Prevalence of burnout among physicians: a systematic review. JAMA 2018;320:1131-50.

Jerome WH Seow, ${ }^{1} M B B S$, Shuxun Lin, ${ }^{1}{ }_{M B B S,}$ MMed, FRCS, Yuet Peng Khor, ${ }^{1}{ }_{M B C h B, M R C S,}$ FRCS, Dennis HW Hey, ${ }^{2}$ MMED, MCI, FRCSEd, Fareed HY Kagda, ${ }^{1} M B B S$, FRCS, FAMS

\footnotetext{
${ }^{1}$ Department of Orthopaedic Surgery, Ng Teng Fong General Hospital, Singapore

${ }^{2}$ Department of Orthopaedic Surgery, National University Hospital, Singapore
}

Address for Correspondence: Dr Jerome Seow, Department of Orthopaedic Surgery, Ng Teng Fong General Hospital, 1 Jurong East Street 21, Singapore 609606.

Email: jerome.seow@mohh.com.sg 